\title{
Philosophiques
}

\section{L'engagement psychologique dans la communication langagière}

\section{Gilles Gauthier}

Volume 16, numéro 1, printemps 1989

URI : https://id.erudit.org/iderudit/027064ar

DOI : https://doi.org/10.7202/027064ar

Aller au sommaire du numéro

Éditeur(s)

Société de philosophie du Québec

ISSN

0316-2923 (imprimé)

1492-1391 (numérique)

Découvrir la revue

Citer cet article

Gauthier, G. (1989). L'engagement psychologique dans la communication langagière. Philosophiques, 16(1), 43-71. https://doi.org/10.7202/027064ar
Résumé de l'article

L'objectif de l'article est de proposer une théorie de l'engagement psychologique dans le langage. C'est-à-dire de présenter une vue systématique de l'implication de la psyché des sujets parlants dans leur accomplissement d'actes de langage ou encore de ce à quoi ils sont assujettis sur le plan psychologique quand ils parlent. Cette théorie a la forme d'une série de thèses chacune relative à un trait spécifique de la performance langagière. Prenant appui sur la philosophie du langage de John Searle et Daniel Vanderveken, elle est élaborée à partir d'une notion originale d'engagement à avoir des états mentaux. 


\title{
L'ENGAGEMENT PSYCHOLOGIQUE DANS LA COMMUNICATION LANGAGIÈRE
}

\author{
par Gilles Gauthier *
}

\begin{abstract}
RÉSUMÉ. L'objectif de l'article est de proposer une théorie de l'engagement psychologique dans le langage. C'est-à-dire de présenter une vue systématique de l'implication de la psyché des sujets parlants dans leur accomplissement d'actes de langage ou encore de ce à quoi ils sont assujettis sur le plan psychologique quand ils parlent. Cette théorie a la forme d'une série de thèses chacune relative à un trait spécifique de la performance langagière. Prenant appui sur la philosophie du langage de John Searle et Daniel Vanderveken, elle est élaborée à partir d'une notion originale d'engagement à avoir des états mentaux.
\end{abstract}

ABSTRACT. The aim of this article is to propose a theory of the psychological commitment in the language. In other words, it is to present a systematic view of how the mental states of the speakers are present in their performance of speech acts, of what are the psychological states to the possession of which the speakers are committed when they speak. This theory is based on several thesis, each of which is about a specific aspect of the performance of speech acts. It is proposed in the perspective of the philosophy of language of John Searle and Daniel Vanderveken on the basis of an original concept of commitment to have mental states.

1. Soit les propos suivants tenus par différents personnages dans différents contextes de communication:

- Un homme politique: «Je promets de faire telle chose... que je ne me crois pas capable de faire».

- La vedette d'une réclame commerciale: "Achetez tel produit... que je ne désire pas vous voir acheter».

* Je tiens à remercier chaleureusement Claude Panaccio pour ses commentaires toujours judicieux sur le contenu de ce texte. 
— Un journaliste: «Reagan a cessé de mentir... mais je ne crois pas qu'il ait déjà menti ».

- Un passant croisé dans la rue : «Le Pape est mort... mais je ne crois pas qu'il ait jamais existé ».

Ces énoncés, même proférés littéralement et dans un contexte normal d'énonciation, ne constituent pas, à strictement parler, des contradictions logiques. Mais les instruments traditionnels des logiques classique et propositionnelle sont inadéquats pour rendre compte de leur déficience formelle. Car ils sont manifestement de quelque façon défectueux. Et leur lacune se répercute jusqu'à la séquence de communication dans laquelle ils s'insèrent qui en devient elle-même déficiente : les auditeurs de ces propos, tout en n'étant pas immédiatement en mesure d'en démontrer l'illogisme au sens strict, ont néanmoins l'intuition que leur structure interne est frappée de défectuosité.

2. Nos quatre exemples ont une particularité commune. Tous sont des doublets constitués d'une première énonciation à laquelle est jointe une dénégation de possession d'un état psychologique, de croyance ou de désir.

L'hypothèse qui sera ici creusée est que leur déficience formelle, qui est bien un type, mais particulier, d'illogisme est relative à la problématique de l'engagement psychologique concomitant à l'accomplissement d'actes de langage. Notre idée est qu'il est possible de systématiser ce à quoi les locuteurs sont liés sur le plan mental ou encore ce qu'il apparaît correct de leur imputer psychologiquement en vertu de leur utilisation du langage et qu'une théorie adéquate de cet engagement psychologique est en mesure d'expliquer la défectuosité inhérente aux énoncés du genre de ceux mentionnés en début de texte, et plus généralement de rendre compte des situations d'illocution et de communication entravées ou bloquées du fait d'une dénégation de possession d'un état mental.

3. La théorie de l'engagement psychologique corrélatif au langage qui sera ici proposée prend racine dans les travaux du philosophe américain John Searle. Depuis déjà plus d'une vingtaine d'années, Searle fignole une théorie philosophique du langage dans laquelle ce dernier est essentiellement considéré du point de vue de l'accomplissement d'actes, reconnus comme étant les unités 
de base de la communication. ${ }^{1} \grave{A}$ cette théorie des actes de langage, Searle a récemment accolé, entre autres choses, une philosophie de l'esprit (ou des états mentaux). ${ }^{2}$ De plus, en collaboration avec Daniel Vanderveken, il a procédé à une formalisation systématique de sa théorie des actes de langage dans une logique illocutoire. ${ }^{3}$

Bien que soit mis en évidence dans la philosophie searlienne le fait que les états psychologiques sont partie prenante à l'accomplissement des actes de langage, Searle et Vanderveken n'ont pas eux-mêmes examiné la problématique de l'engagement psychologique telle que nous l'abordons. Les concepts qui pour ce faire seront ici développés sont donc originaux en dépit de leur inspiration franchement searlienne. Il arrive par ailleurs que leur considération fasse apparaître l'imprécision ou l'inaptitude de certains développements de la philosophie searlienne.

Une dernière remarque préliminaire s'impose. Le texte qu'on va lire n'a absolument pas de prétention d'ordre psychologique et encore moins ontologique. Il n'a pas pour but de décrire les mécanismes de l'esprit humain dont il aurait au préalable reconnu ou démontré l'existence effective. Son objectif est plutôt de nature strictement logique : déterminer ce à quoi l'utilisation du langage engage et par voie de conséquence contraint sur le plan mental.

4. Notre investigation de la problématique de l'engagement psychologique adopte comme point de départ l'ensemble de la philosophie searlienne. Nous faisons donc nôtres les principales caractérisations qui y sont fournies du langage, considéré sous l'angle de l'accomplissement d'actes, et de la psyché, conçue comme étant constituée d'états mentaux, pour certains intentionnels.

Selon Searle et Vanderveken, les unités minimales de la communication linguistique sont les actes de langage appelés «illocutoires » :

1. Searle, J.R., Speech Acts. An Essay in the Philosophy of Language., Cambridge: Cambridge University Press, 1969, 203 p. ; Expression and Meaning. Studies in the Theory of Speech Acts., Cambridge : Cambridge University Press, 1979, 187 p.

2. Searie, J.R., Intentionality. An Essay in the Philosophy of Mind., Cambridge: Cambridge University Press, 1983, 278 P.

3. Searle, J.R. et Vanderveken, D., Foundations of Illocutionary Logic, Cambridge Cambridge University Press, 1985, 227 p. 
The minimal units of human communication are speech acts of a type called illocutionary acts. ${ }^{4}$

Les actes illocutoires, tels la promesse, l'ordre, l'assertion, l'excuse, etc., sont ce qui est fondamentalement produit par l'emploi du langage. En d'autres termes, les actes illocutoires ont trait aux différentes fonctions possibles exerçées par l'utilisation d'éléments linguistiques.

Ils sont constitués de deux composants abstraits : une force illocutoire et un contenu propositionnel. La force illocutoire, pour la caractériser informellement, est ce en vertu de quoi l'énonciation (littérale, dans un contexte approprié) d'un énoncé ou d'une phrase constitue l'accomplissement d'un acte de langage. Quant au contenu propositionnel d'un acte de langage, il a trait, disons, à «ce dont il y est question », sous une certaine force illocutoire ; c'està-dire cette partie de la «réalité » sur laquelle porte l'acte de langage. Selon Searle et Vanderveken, un acte de langage a ainsi pour contenu une représentation d'un état de choses sous la forme d'une proposition.

La réussite des actes illocutoires est sujette à différentes conditions. Deux de ces conditions retiennent davantage l'attention.

D'abord une condition de sincérité. D'après Searle et Vanderveken, un locuteur qui accomplit un acte de langage donné exprime un certain état psychologique et cet état mental constitue la condition de sincérité de son acte. Par exemple, la condition de sincérité d'une assertion est la croyance à la réalité de ce qui est affirmé, celle d'une promesse est l'intention d'effectuer la chose promise et celle d'un ordre le désir que l'allocutaire l'exécute.

Outre cette condition de sincérité, un acte illocutoire tombe également sous le coup d'une condition préparatoire. Par exemple, l'accomplissement d'un ordre a pour disposition particulière de cette sorte que l'allocutaire soit capable de l'effectuer. De même, l'acte de déclarer ouverte une assemblée exige que le locuteur ait le pouvoir ou l'autorité pour que ce qu'il proclame devienne le cas. Telle que considérée dans la philosophie searlienne, la condition préparatoire d'un acte de langage est en fait un état de choses que

4. Fondations of Illocutionary Logic, p. I. 
le locuteur suppose être actualisé quand il accomplit l'acte. Elle consiste donc ou a trait à une présupposition dite «illocutoire» parce qu'elle fait partie, comme la condition de sincérité d'ailleurs, de la force illocutoire de l'acte de langage. Les présuppositions illocutoires sont des relations ternaires entre les locuteurs, leurs actes de langage et des propositions : le locuteur qui accomplit un acte de langage présuppose la vérité de la proposition représentant l'état de choses constituant la condition préparatoire de l'acte.

Les présuppositions illocutoires se distinguent de ces autres présuppositions plus classiques que sont les présuppositions propositionnelles. Traditionnellement, une présupposition propositionnelle est considérée comme étant elle-même une proposition dont la vérité est «impliquée » (dans le sens d' « exigée») par une première proposition. Par exemple, la proposition «Pierre est revenu d'Europe» implique que soit vraie la proposition «Pierre était en Europe ».

Dans la mesure où les propositions peuvent être abordées strictement pour elles-mêmes, il est possible, dans une perspective logico-sémantique, d'ainsi caractériser les présuppositions propositionnelles comme étant des relations binaires entre propositions. Il est par ailleurs également possible, puisque - tel que conçu dans la philosophie searlienne - le contenu de représentation des actes de langage consiste précisément en une proposition, de considérer que les présuppositions propositionnelles sont plutôt, à l'image des présuppositions illocutoires, des relations ternaires entre des locuteurs, des actes de langage et des propositions. Suivant ce point de vue plus pragrnatique, un locuteur qui accomplit un acte de langage doit supposer qu'est actualisé l'état de choses correspondant à une proposition dont la vérité est présupposée par la proposition constituant le contenu de représentation de l'acte. Par exemple, le locuteur qui donne l'ordre à Pierre de revenir d'Europe " pose» - dans la visée de son désir, pourrait-on dire - l'état de choses du retour prochain d'Europe de Pierre et présuppose celui du séjour actuel de Pierre en Europe.

Sujets à des conditions d'accomplissement, les actes de langage sont évaluables en termes de réussite ou d'échec. Il peut par ailleurs arriver, toujours selon Searle et Vanderveken, qu'un acte de langage soit effectué avec succès mais de façon défectueuse. Il en 
est ainsi si l'état de choses correspondant à une condition préparatoire ou à une présupposition propositionnelle de l'acte n'est pas actualisé, en dépit du fait que le locuteur présuppose que c'est le cas. Ou encore si n'est pas remplie la condition de sincérité, c'està-dire si le locuteur ne possède pas l'état psychologique qu'il exprime en accomplissant l'acte. Dans l'élaboration de notre théorie de l'engagement psychologique langagier, seul l'accomplissement réussi et non-défectueux des actes de langage sera considéré.

De même, ne seront ici considérés que les actes de langage les plus usuels que nous appelons «standards», à savoir les actes de langage ayant un contenu propositionnel constitué d'une référence et d'une prédication. Sont ainsi exclus de notre propos des actes de langage comme les affirmations existentielles dont le contenu propositionnel ne comporte pas une référence et plus généralement tous les actes de langage qui pourraient de quelque façon s'avérer propositionnellement déficients.

Une dernière notion de la philosophie searlienne va nous être utile: l'incompatibilité illocutoire. Deux actes de langage sont (relativement) incompatibles s'il ne peuvent être simultanément accomplis, c'est-à-dire s'il n'est pas possible à un locuteur de les accomplir tous deux dans le même contexte d'énonciation. Les exemples les plus patents d'actes incompatibles sont un acte de langage donné et sa dénégation illocutoire. Par exemple, un locuteur ne peut pas dans le même contexte d'énonciation simultanément accomplir l'assertion « La terre est ronde » et la dénégation «Je n'affirme pas que la terre est ronde».

5. La notion fondamentale retenue aux fins de la présente recherche est celle d'engagement à avoir des états psychologiques. Ainsi que nous l'entendons, elle dénote une relation ternaire entre un sujet parlant, son accomplissement d'un acte de langage et un état mental. Nous soumettons - ce ne peut être à ce stade qu'une proposition heuristique dont les exemplifications seront plus loin fondées - qu'en accomplissant un acte de langage donné, un locuteur se trouve ipso facto tenu à la possession d'un certain nombre d'états psychologiques précis.

L'idée que la psyché des locuteurs soit à ce point impliquée dans leur accomplissement d'actes de langage n'est pas étrangère à 
la philosophie searlienne. Ainsi que nous venons de le voir, Searle et Vanderveken conçoivent clairement que les états psychologiques sont partie prenante à l'accomplissement d'actes de langage sous la forme de conditions de sincérité. Ils reconnaissent même expressément qu'il n'est pas possible à un locuteur d'accomplir des actes de langage sans être par le fait même commis de quelque façon sur le plan mental.

Searle et Vanderveken mettent ainsi explicitement de l'avant une notion d'engagement à avoir des états mentaux, ou plutôt deux notions distinctes de cet engagement psychologique. Ils introduisent cette considération relativement au projet d'élaboration d'une logique des états psychologiques, plus précisément des attitudes propositionnelles. Cette logique, parallèle sous plusieurs aspects à la logique illocutoire, aurait pour objectif essentiel de fournir une caractérisation formelle des états mentaux. Quelquesunes des questions dont elle aurait à traiter sont les suivantes:

... What is the logical form of the various psychological states? What are the necessary and sufficient conditions for having each type of psychological state? What are the logical relations between the various types? In particular when does the possession of one psychological state necessarily involve the possession of another? Similarly, when does the expression of one psychological state commit the speaker to the possession of another?...5 (C'est moi qui souligne).

Les concepts searliens d'engagement à avoir des états mentaux ont trait, l'un à la possession, l'autre à l'expression préalable d'un premier état psychologique. En fait, ils dénotent chacun une relation ternaire différente.

La première est celle qui peut être établie entre un sujet parlant, sa possession d'un état mental et un second état psychologique qu'il serait engagé à avoir du fait de cette possession. Appelons cette notion l'«engagement (POSS)» afin de bien marquer qu'il est relatif à la possession préalable d'un premier état mental. Searle et Vanderveken donnent comme exemple de cette forme d'engagement psychologique la croyance en $\mathrm{P} \& \mathrm{Q}$ dont la possession engage à avoir la croyance en $Q$.

S. Ibid., p. 102. 
Le second concept d'engagement psychologique mis en évidence dans la philosophie searlienne dénote la relation ternaire entre un sujet parlant, son expression d'un état mental et un état à la possession duquel le locuteur serait tenu du fait de cette expression psychologique préalable. L'état mental faisant l'objet de l'engagement peut ne pas être lui-même exprimé :

... the expression of a psychological state in the performance of the illocution can commit (the speaker) to having a state he has not expressed. Thus, for example, a speaker who expresses a belief that $P$ and a belief that if $\mathrm{P}$ then $\mathrm{Q}$ is committed to having the belief that $\mathrm{Q} .{ }^{6}$

Par ailleurs, la relation d'engagement psychologique relatif à l'expression d'un premier état mental est réflexive : elle vaut (et même d'abord) pour l'état qui est exprimé. Un locuteur est ainsi engagé à avoir l'état mental qu'il exprime:

The expression of a state commits the speaker to having that state... ${ }^{7}$

C'est cette expression d'un état psychologique qui constitue la condition de sincérité de l'accomplissement d'un acte de langage.

De façon à le distinguer de la notion d'engagement (POSS) et à mettre en évidence le fait qu'il soit relatif à l'expression préalable d'états mentaux, donnons au second concept d'engagement psychologique l'appellation d' " engagement (EXPR)».

Les concepts searliens d'engagement (POSS) et d'engagement (EXPR) ne se prêtent pas à une investigation correcte de la problématique de l'engagement psychologique dans le langage parce qu'ils ne suffisent pas, même à deux, à rendre compte de toutes les façons dont la performance langagière engage les locuteurs à la possession d'états mentaux.

D'abord, l'engagement (POSS) n'a rien à voir, intrinsèquement, avec le langage: c'est indépendamment du fait et même de la faculté de parler que les sujets ont des états et que cette possession engage à la possession d'autres états. Par conséquent, on aura beau avoir établi systématiquement à quels autres états psychologiques la possession d'un premier état engage, on n'aura en aucune façon,

6. Ibid., p. 19 .

7. Ibid., p. 19 
de ce seul fait, avancé quoi que ce soit au sujet de l'engagement psychologique langagier. Il faut par ailleurs considérer qu'un locuteur qui accomplit un acte de langage peut justement être engagé à avoir un état psychologique. S'il se trouve que la possession de ce dernier état engage à avoir un autre état mental, le locuteur sera évidemment contraint à cet engagement. Sa performance langagière l'engagera alors, transitivement pour ainsi dire, à avoir un second état mental du fait qu'elle l'engage d'abord à avoir un premier état. Le concept d'engagement (POSS) pourrait ainsi s'avérer utile au traitement tout à fait intégral de la problématique de l'engagement psychologique mais pas uniquement de luimême; ce ne le serait qu'en seconde instance, sur la base d'un engagement d'ordre langagier plus fondamental, celui qui fait précisément l'objet de la présente recherche.

Pas davantage que le concept d'engagement (POSS), le concept d'engagement (EXPR) mis en évidence dans la philosophie searlienne ne peut convenablement contribuer au traitement adéquat de la problématique de l'engagement psychologique dans le langage. Il met bien en jeu la performance langagière mais demeure de portée trop restreinte. En effet, suivant (le seul) concept d'engagement (EXPR), l'accomplissement d'un acte de langage pourrait engager le locuteur à avoir un ou des état(s) psychologique(s) uniquement en raison du fait qu'il exprime d'abord un premier état mental. Certes, cela peut bien être le cas : comme Searle et Vanderveken le donnent en exemple, l'expression de la croyance que $\mathrm{P}$ et de la croyance que si $\mathrm{P}$ alors $\mathrm{Q}$ engage le locuteur à avoir la croyance que $Q$. Ce n'est cependant pas la seule façon par laquelle un locuteur peut être engagé à la possession d'états mentaux ; il existe d'autres voies que celle de l'expression psychologique préalable suivant lesquelles un locuteur peut contracter un engagement à la possession d'états psychologiques. Il apparaît par exemple - pour le moment, considérons intuitivement la chose à titre strictement hypothétique - que le locuteur qui accomplit un acte d'ordre doit croire que l'allocutaire est capable d'effectuer l'action qu'il lui ordonne de faire. L'engagement à avoir cette croyance ne découle pas de l'expression d'un autre état mental dans l'accomplissement de l'acte d'ordre. Que cet engagement soit effectif, cela reste bien sûr à être par ailleurs démontré. Mais la considération exclusive du concept d'engagement (EXPR) ne permettrait même pas de le 
repérer. Autrement dit, la réduction de la problématique de l'engagement psychologique à ce concept d'engagement (EXPR) par sa seule prise en compte comporte le risque d'occulter des pans entiers de la problématique.

Aussi, afin de considérer adéquatement cette question, faut-il proposer un nouveau concept d'engagement à avoir des états mentaux qui à la fois soit d'ordre proprement langagier, contrairement au concept d'engagement (POSS), et dépasse tout en l'englobant le concept d'engagement (EXPR). Ce concept, c'est celui qu'on peut appeler l'«engagement (PERF)»: l'engagement à avoir un état psychologique du fait (général) de la performance langagière. Il dénote donc la relation ternaire entre un sujet parlant, son accomplissement d'un acte de langage et un état mental que ce faisant il est engagé à avoir. Comparativement à l'engagement (POSS), l'engagement (PERF) restreint l'engagement à la possession d'états psychologiques à l'utilisation du langage ; comparativement à l'engagement (EXPR), il ne le limite pas abusivement à une seule de ses causes possibles tout en la conservant. Car, bien sûr, la relation entre un sujet parlant, son expression (langagière) d'un premier état psychologique et un second état mental est comprise dans la relation plus globale entre ce même sujet parlant, son accomplissement d'un acte de langage - lieu d'expression psychologique - et le deuxième état mental. D'une plus grande extension, le concept d'engagement (PERF) comporte l'avantage par rapport au concept d'engagement (EXPR) de permettre l'identification éventuelle d'états mentaux qu'un locuteur pourrait être engagé à avoir du fait de son accomplissement d'un acte de langage autrement qu'en vertu de son expression psychologique.

Chercher à investiguer la problématique de l'engagement psychologique dans le langage en prenant comme point de départ le concept d'engagement (PERF), c'est adopter la considération primitive qu'en accomplissant un acte de langage donné, un locuteur se trouve ipso facto, vraisemblablement suivant diverses voies, tenu à la possession d'un certain nombre d'états psychologiques précis. Reste bien sûr à fonder les exemplifications de cette proposition heuristique. 
6. La fécondité théorique du concept d'engagement (PERF) dans le traitement de la problématique de l'engagement psychologique apparaît immédiatement en ce qu'il permet de poser un diagnostic rapide et précis sur les doublets constitués d'une dénégation de possession d'un état mental joint à une première énonciation du genre de ceux donnés en exemples en début d'article. Le concept d'engagement (PERF) permet en effet de cerner le type de déficience formelle qui les affecte.

Précisons d'abord à cet égard un trait général relatif à l'engagement à avoir un état psychologique (quel que soit le concept qu'on en retienne) : il n'implique pas la possession effective de cet état mental. Au contraire, il est en effet tout à fait possible à un locuteur d'être engagé à avoir un état psychologique sans qu'il le possède réellement. C'est d'ailleurs, pour une bonne part tout au moins, en fonction de cette possibilité de ne pas avoir un état psychologique à la possession duquel on est engagé que le mensonge et autres tromperies d'illocution et de communication sont possibles et organisés. Celui qui fait une promesse - pour reprendre l'exemple maintenant classique de la philosophie searlienne - peut ne pas avoir l'intention de faire la chose promise même s'il est engagé à avoir cette intention. Sa promesse est alors insincère. Cette insincérité origine précisément du fait que le locuteur n'a pas l'intention qu'il est engagé à avoir en promettant.

Si un locuteur peut ainsi ne pas avoir effectivement un état psychologique qu'il est engagé à avoir, il ne peut pas logiquement affirmer qu'il n'a pas l'état mental en question. Le locuteur qui oserait avouer qu'il n'a pas l'intention d'effectuer l'action qu'il vient de promettre d'effectuer non seulement dévoilerait son insincérité mais compromettrait également de façon irrémédiable l'efficace de son discours. Autrement dit, il y a incompatibilité (relative) entre un acte de langage engageant à la possession d'un état psychologique et la dénégation de possession de cet état mental. C'est-à-dire que les deux actes de langage ne peuvent être simultanément accomplis dans le même contexte d'énonciation. Searle et Vanderveken reconnaissent explicitement cette incompatibilité dans le cas de l'état psychologique exprimé, à titre de condition de sincérité, dans l'accomplissement d'un acte de langage, comme par exemple l'intention dans l'accomplissement d'une promesse : 
... an assertion that one does not intend to carry out a future course of action is relatively incompatible with a promise to carry out that course of action; that is because a promise has the sincerity condition that the speaker intends to do what he commits himself and an assertion that one does not have a certain psychological state is relatively inconsistant with the expression of that psychological state. ${ }^{8}$

L'incompatibilité ici signalée est relative au fait que l'état mental est exprimé à l'occasion de l'accomplissement de l'acte. Autrement dit, c'est eu égard à ce que nous avons appelé le concept d' « engagement (EXPR)» que l'incompatibilité entre un acte de langage et la dénégation de possession d'un état psychologique est introduite dans la philosophie searlienne. C'est encore plus clair dans cet autre passage :

The fact that the expression of the psychological state is internal to the performance of the illocution is shown by the fact that it is paradoxal to perform an illocution and to deny simultaneously that one has the corresponding psychological state. Thus, one cannot say «I promise to come but I do not intend to come », «I order you to leave but I don't want you to leave», "I apologize but I am not sorry», etc. And this incidentally explains Moore's paradox that one cannot say consistently «It is raining but I don't believe that it is raining " even though the proposition that it is raining is consistent with the proposition that I do not believe that it is raining. The reason for this is that when one performs the speech act one necessarily expresses the sincerity condition, and thus to conjoin the performance of the speech act with the denial of the sincerity condition would be to express and to deny the presence of one and the same psychological state.

Non seulement l'incompatibilité entre la dénégation de possession d'un état mental et un acte de langage est-elle ici de nouveau présentée relativement à l'expression de l'état dans l'accomplissement de l'acte, mais elle est au surplus considérée comme étant la preuve ou le test de l'expression de l'état psychologique dans l'accomplissement de l'acte de langage. Le fait qu'il soit " paradoxal » d'accomplir un acte de langage et de dénier avoir tel état psychologique - que les deux actes ne peuvent être simultanément accomplis, qu'ils soient donc incompatibles - suffirait à la démonstration que cet état est exprimé dans l'accomplissement de l'acte. C'est ainsi par une généralisation du paradoxe de Moore 
que Searle et Vanderveken prétendent rendre compte de l'expression psychologique langagière.

Or, il est possible de soutenir que l'incompatibilité qu'à cette fin ils mettent en cause n'a rien à voir véritablement avec l'expression psychologique mais est plutôt relative, plus généralement, à l'engagement à avoir des états mentaux du fait de l'accomplissement d'actes de langage et qu'elle ne peut donc servir de «preuve » ou de «test» à l'expression d'un état mental dans le langage. Bref, l'incompatibilité dont il est ici question n'est pas relative au concept d'engagement (EXPR) mais bien à celui d'engagement (PERF).

Reconsidérons les actes de promesse «Je promets de venir» et d'assertion «Il pleut donnés en exemple, entre autres, par Searle et Vanderveken. Qu'advient-il si nous mettons entre parenthèses la question de savoir si les états psychologiques d'intention de venir et de croyance qu'il pleut y sont respectivement exprimés? Autrement dit, quelle conséquence entraîne la décision de réserver notre jugement sur le fait que l'accomplissement des actes de promesse et d'assertion soient effectivement ou non le lieu d'expression de ces états mentaux? Il est remarquable que cette réserve n'enlève rien et n'entraîne aucune défectuosité dans la suite de l'argument relatif à l'incompatibilité entre les actes et la dénégation de possession des états. Simplement en admettant généralement que l'accomplissement des actes engage à la possession des états, laissant donc en suspens la question de savoir si les états sont exprimés lors de l'accomplissement des actes, s'impose le constat qu'un locuteur ne peut simultanément promettre de venir et prétendre ne pas avoir cette intention ou affirmer qu'il pleut et ne pas avoir la croyance qu'il pleut. Il est paradoxal de dénier posséder des états mentaux en accomplissant des actes de langage indépendamment du fait que les états soient exprimés ou non dans les actes.

Ainsi, c'est donc parce qu'un locuteur est engagé à avoir un état psychologique dans son accomplissement d'un acte de langage et non pas parce qu'il exprime cet état quand il accomplit l'acte qu'il y a incompatibilité entre cet acte et la dénégation de possession de l'état psychologique. Il se trouve que le locuteur qui exprime un état mental à l'occasion de son accomplissement d'un acte de 
langage est engagé à la possession de cet état. C'est cet engagement à avoir l'état et non son expression qui rend paradoxale la dénégation de possession de l'état, qui fait que cette dénégation et l'acte de langage sont incompatibles.

Que cette incompatibilité soit relative non pas à l'expression d'états psychologiques mais plutôt à l'engagement à la possession de ces états est encore indiqué par le fait qu'elle s'applique à des états mentaux qu'un locuteur est engagé à avoir du fait de son accomplissement d'actes de langage mais qu'il n'exprime manifestement pas en accomplissant ces actes. Considérons deux exemples. Le premier est donné par Searle et Vanderveken eux-mêmes quand ils introduisent ce que nous avons nommé leur concept d' "engagement (EXPR)»: l'expression des croyances que $P$ et que si $\mathrm{P}$ alors $\mathrm{Q}$ dans l'accomplissement d'un acte de langage engage à avoir cette autre croyance que $Q$. Le second exemple est celui-là même que nous avons proposé pour illustrer notre concept d'engagement (PERF) : l'accomplissement d'un ordre engage le locuteur à avoir la croyance que l'allocutaire est capable d'accomplir l'action requise. Exactement comme dans le cas d'un état exprimé, il est tout à fait paradoxal pour un locuteur ayant exprimé les croyances que $\mathrm{P}$ et que si $\mathrm{P}$ alors $\mathrm{Q}$ de dénier avoir la croyance que $\mathrm{Q}$ ou pour un locuteur qui donne un ordre de dénier avoir la croyance que l'allocutaire est capable de l'effectuer. Il y a incompatibilité (relative) entre les actes de langage où sont exprimées les croyances que $\mathrm{P}$ et que si $\mathrm{P}$ alors $\mathrm{Q}$ et la dénégation de possession de la croyance que $Q$ ainsi qu'entre un ordre et la dénégation de possession de la croyance que l'allocutaire a la capacité de faire l'action ordonnée.

Si donc l'incompatibilité entre la dénégation de possession d'un état psychologique et un acte de langage s'applique dans le cas des états mentaux exprimés non pas parce qu'ils sont exprimés mais plutôt parce que les locuteurs sont engagés à leur possession et qu'elle vaut également pour les états qui ne sont pas exprimés, elle ne peut être invoquée pour démontrer le fait que des états psychologiques sont bien exprimés dans l'accomplissement des actes de langage. L'incompatibilité entre un acte de langage et la dénégation de possession d'un état mental ne peut servir de preuve ou de test à l'expression psychologique langagière ainsi que Searle et Vanderveken le souhaiteraient. Relative tout autant à des 
états mentaux qui ne font pas l'objet d'une expression, cette incompatibilité n'est pas en mesure de discriminer les états exprimés des états non exprimés et donc non plus de démontrer que tel état psychologique est exprimé plutôt que non dans l'accomplissement d'un acte de langage. De même, quand elle s'applique à des états exprimés, la notion d'incompatibilité ne se trouve pas, par généralisation du paradoxe de Moore, à rendre manifeste qu'un état mental est exprimé dans l'accomplissement d'un acte de langage mais plus généralement que l'accomplissement de l'acte engage le locuteur à la possession de cet état psychologique.

L'examen qui précède de l'incompatibilité entre la dénégation de possession d'un état mental et un acte de langage amène par ailleurs encore de l'eau au moulin à notre proposition de recourir au concept d'engagement (PERF) plutôt qu'à celui d'engagement (EXPR) pour mener l'investigation de la problématique de l'engagement psychologique. Car si l'incompatibilité en question est bien relative non pas à l'expression d'états psychologiques dans l'accomplissement de l'acte mais plus précisément au fait que celle-ci engage le locuteur à avoir des états psychologiques, il suit que la notion d'engagement (EXPR) est un concept tout à fait vide du point de vue de notre recherche : l'incompatibilité entre un acte de langage et la dénégation de possession d'un état fait clairement voir que ce n'est pas parce qu'il est exprimé ou du fait de son expression que le locuteur est engagé à avoir cet état quand il accomplit l'acte. Pour le cas où elle est effective, l'expression psychologique est secondaire, non pertinente à l'engagement à avoir des états mentaux et à l'incompatibilité mettant en jeu une dénégation de possession d'un tel état psychologique.

Eu égard à la problématique de l'engagement psychologique dans le langage, la notion d'incompatibilité entre la dénégation de possession d'un état mental et un acte de langage comporte un avantage heuristique de grande valeur : elle permet de reconnaître l'incongruité qu'il y a pour un locuteur de nier avoir l'état psychologique que son accomplissement d'un acte de langage l'engage à avoir. Elle dévoile ainsi l'illogisme des doublets du type de nos exemples du début : c'est précisément parce que leur second terme qui consiste en la dénégation d'un état mental est incompatible avec leur premier terme qu'ils sont frappés de déficience formelle. 
Leur défectuosité s'explique par le fait qu'il est inconsistant ou paradoxal pour un locuteur d'accomplir l'acte de langage faisant l'objet du premier terme du doublet tout en niant avoir l'état psychologique spécifié dans le second terme.

La notion d'incompatibilité a ici strictement une fonction de diagnostic. Elle établit la nature défectueuse de nos doublets sans en rendre compte. Il reste à déterminer la raison même de cette incompatibilité, c'est-à-dire expliquer en quoi le premier terme de nos doublets engage le locuteur à avoir l'état mental faisant l'objet de la dénégation de possession qui lui est accolée. Plus généralement, il faut théoriser l'engagement psychologique concomitant au langage, c'est-à-dire systématiser l'engagement à avoir un ensemble d'états mentaux corrélatif à l'accomplissement d'actes de langage et de ce fait rendre compte de l'incompatibilité entre l'accomplissement de ces actes et la dénégation de possession de ces états.

7. La théorie de l'engagement psychologique dans le langage que nous proposons prend comme considération primitive le concept d'engagement (PERF) de préférence aux concepts searliens d'engagement (POSS) et d'engagement (EXPR) en raison de sa plus grande précision et de sa plus grande portée. Afin d'explorer correctement et exhaustivement la problématique de l'engagement d'ordre mental dans la performance langagière, il importe de laisser ouverte la possibilité qu'un locuteur puisse être engagé à avoir un état psychologique autrement que parce qu'il possède ou exprime d'abord cet état ou un autre état mental. Seul le concept d'engagement (PERF) est suffisamment large pour rendre compte des cas d'engagement à avoir un état mental indépendamment de la possession ou de l'expression psychologique préalable.

Soit, par exemple, l'idée - plus haut intuitivement évoquée et que nous chercherons sous peu à établir plus formellement - , que l'accomplissement d'un acte d'ordre engage le locuteur à avoir la croyance que l'allocutaire est en mesure d'effectuer l'action commandée. Comme cette croyance peut ne pas être possédée et n'est certainement pas exprimée par le locuteur quand il donne son ordre et comme également l'engagement à avoir cette croyance ne découle pas non plus de la possession ou de l'expression d'un autre état mental, les concepts d'engagement (POSS) et d'engagement (EXPR) se révèlent tout à fait inaptes à déceler et à 
expliquer l'engagement psychologique considéré. Ces deux concepts searliens sont d'une portée trop limitative ${ }^{10}$ qui les empêche de traiter adéquatement des cas d'engagement psychologique autres que ceux qui résultent de la possession ou de l'expression d'un état mental. L'engagement à avoir la croyance que l'allocutaire est capable d'exécurer un ordre procède non pas d'une telle possession ou expression préalable mais plutôt de l'accomplissement, plus précisément d'un autre aspect différent de l'accomplissement de l'acte d'ordre. Pour le repérer et en rendre compte, il faut recourir au concept à la fois plus englobant et plus spécifique d'engagement (PERF) à la possession d'états mentaux.

La théorie de l'engagement psychologique dans le langage qu'il est possible de construire autour de ce concept d'engagement (PERF) a pour objectif de déterminer exhaustivement ce à quoi consentent les locuteurs sur le plan mental du fait de leur performance langagière. Cette tâche s'avère possible parce que, suivant l'économie de la philosophie searlienne, l'utilisation du langage est non pas aléatoire mais réglée, organisée. Il est possible d'identifier les états psychologiques à la possession desquels un locuteur est engagé (PERF) en considérant les conditions d'accomplissement et les présuppositions propositionnelles des actes de langage. C'est en vertu de ces traits structuraux qu'accomplissant un acte de langage un locuteur contracte un engagement à avoir certains états mentaux.

Cela veut dire que, sur le plan heuristique, c'est eu égard aux conditions d'accomplissement et aux présuppositions propositionnelles que le concept d'engagement (PERF) est opératoire: chacune de ces conditions et présuppositions entraîne pour le locuteur l'engagement à la possession d'un ou de plusieurs états psychologiques bien spécifiques. La théorie de l'engagement psychologique dans la communication langagière prend ainsi la forme de thèses successives relatives aux conditions de l'accomplissement des actes de langage et à leurs présuppositions propositionnelles.

10. Pour les fins, évidemment, de l'élucidation de la problématique de l'engagement psychologique dans le langage; peut-être pas par ailleurs pour l'usage que Searle et Vanderveken en font dans leur logique illocutoire. 
La première de ces thèses est la suivante.

THÈSE A : En accomplissant un acte de langage, un locuteur est engagé à avoir l'état psychologique constituant la condition de sincérité de l'acte.

La condition d'accomplissement des actes de langage qui apparaît manifestement comme la plus pertinente à la problématique de l'engagement psychologique est certes la condition de sincérité puisqu'elle porte précisément sur un état mental dont (incidemment) l'expression serait inhérente à l'accomplissement de l'acte.

Notre thèse $A$ stipule que le locuteur se trouve engagé à la possession de cet état mental qui est impliqué à titre de condition de sincérité dans son accomplissement d'un acte de langage. Par exemple, un locuteur est engagé à a voir la croyance en la teneur de son assertion, l'intention d'effectuer la chose qu'il promet de faire et le désir que l'allocutaire exécute l'ordre qu'il lui donne.

L'engagement à la possession de l'état psychologique constituant la condition de sincérité d'un acte de langage fait en sorte qu'accomplissant l'acte, un locuteur ne peut dénier avoir l'état mental. L'acte de langage est incompatible avec la dénégation de possession de l'état: ils ne peuvent être simultanément accomplis dans le même contexte d'énonciation. Il serait paradoxal qu'un locuteur fasse une assertion et nie avoir la croyance en sa teneur, fasse une promesse et nie avoir l'intention de la remplir ou donne un ordre et nie avoir le désir que l'allocutaire l'exécute.

En vérité, compte tenu de l'identification, telle que posée dans la philosophie searlienne, de la condition de sincérité à l'expression d'un état psychologique, la thèse $A$ apparaît presque triviale. D'ailleurs, Searle et Vanderveken eux-mêmes en reconnaissent explicitement le bien-fondé : ( The expression of a state commits the speaker to having that state...") ${ }^{11}$

La condition de sincérité n'est toutefois pas la seule relativement à laquelle un locuteur est engagé à la possession d'un état mental. D'autres conditions d'accomplissement des actes de langage ont également cette conséquence et donc donnent lieu à d'autres thèses d'engagement psychologique, plus originales que la thèse $A$.

11. Ibid., p. 19. 
Avant de les considérer, une dernière remarque s'impose au sujet de cette première thèse. Elle prend appui sur les deux concepts searliens de condition de sincérité et d'expression psychologique. Or, cette dernière notion n'est pas très claire. En fait, on ne sait trop ce que c'est précisément qu'exprimer un état psychologique. Searle et Vanderveken ne fournissent pas de caractérisation véritable de l'expression psychologique mais se contentent de la proposer de manière toute métaphorique:

... people may be said to "express», "manifest ", or " give vent to » feelings - whether or not they actually have the feeling that they express... 12

Il serait souhaitable de préciser plus explicitement en quoi consiste l'expression d'un état mental parce que dans l'accomplissement d'un acte de langage, ainsi que Searle et Vanderveken le reconnaissent eux-mêmes, un locuteur peut être engagé à avoir des états psychologiques qu'il exprime et d'autres qu'il n'exprime pas. Que fait-il de plus avec ou de l'état mental qu'il exprime qu'il ne fait pas eu égard à un état qu'il est engagé à avoir mais sans l'exprimer ? Nous n'avons pas pour notre part à donner réponse à cette question. Car, ainsi que nous l'avons déjà établi, le fait qu'un état soit exprimé n'est pas pertinent à cet autre fait différent qu'un locuteur soit engagé à l'avoir quand il accomplit un acte de langage. Voilà pourquoi notre thèse $A$ ne fait pas mention de l'expression psychologique mais plus simplement et généralement de la condition de sincérité de la performance langagière.

THÈSE B : En accomplissant un acte de langage, un locuteur est engagé à avoir la croyance en l'état de choses constituant la condition préparatoire de l'acte.

Un locuteur est engagé à avoir la croyance en un état de choses correspondant à une présupposition illocutoire de son acte de langage, c'est-à-dire un état de choses qu'il présuppose être actualisé à titre de condition préparatoire de l'acte. Ainsi, - tel que nous l'avons intuitivement présumé - un locuteur qui donne un ordre est bel et bien engagé à avoir la croyance en la capacité de l'allocutaire d'exécuter l'action ordonnée parce que cette capacité 
constitue une condition préparatoire de l'accomplissement de l'acte d'ordre.

Suivant notre thèse $B$, le locuteur ne peut accomplir un acte de langage et dénier avoir l'état mental de croyance en l'état de choses constituant la condition préparatoire de cet acte ou correspondant à sa présupposition illocutoire. L'acte de donner un ordre et la dénégation de possession de la croyance que l'allocutaire est capable de l'exécuter sont donc incompatibles : leur accomplissement simultané serait inconsistant ou paradoxal.

THÈSE C: En accomplissant un acte de langage, un locuteur est engagé à avoir la croyance en l'état de choses correspondant à la proposition qui est une présupposition propositionnelle de l'acte.

En vertu de la thèse $B$, une présupposition illocutoire a trait à un état psychologique de croyance à la possession duquel un locuteur est commis. Si une présupposition illocutoire donne ainsi lieu à un engagement à avoir un état de croyance qu'elle constitue, il n'y a aucune raison pour que ce ne soit pas également le cas pour une présupposition propositionnelle. La thèse $C$ stipule que le locuteur se trouve à être engagé à la possession de l'état mental de croyance en l'état de choses correspondant à une présupposition propositionnelle d'un acte de langage. Le locuteur qui, par exemple, donne l'ordre à Pierre de revenir d'Europe est engagé à avoir la croyance que Pierre est en Europe. Si la proposition constitutive du contenu de représentation d'un acte de langage implique la vérité d'une seconde proposition, le locuteur qui accomplit cet acte est engagé à l'égard de la vérité de cette présupposition propositionnelle; il est engagé à la possession de la croyance en l'état de choses auquel elle correspond. Le locuteur qui donne l'ordre à Pierre de rentrer d'Europe ne peut ainsi, sans inconsistance, simultanément affirmer ne pas avoir la croyance que Pierre est en Europe. Un acte de langage est incompatible avec la dénégation de possession de l'état de croyance en l'état de choses correspondant à une présupposition propositionnelle de l'acte.

Il est par ailleurs une présupposition propositionnelle d'une nature particulière commune à tous les actes de langage, à tout le moins à tous les actes de langage standards (c'est-à-dire les actes ayant un contenu propositionnel constitué d'une référence et 
d'une prédication). Cette présupposition est la proposition représentant comme étant existante l'entité à laquelle il est fait référence dans l'acte de langage. Par exemple, l'affirmation "Jean fume beaucoup» et l'ordre «Paul, viens ici!» ont respectivement pour présupposition propositionnelle particulière les propositions existentielles «Jean est (existe) » et «Paul est (existe) ».

L'argumentation qui précède au sujet des présuppositions propositionnelles vaut évidemment pour cette présupposition particulière. Sa nature tout à fait spéciale, relative entre autres choses au fait qu'elle soit commune à tous les actes de langage, rend souhaitable la formulation d'une thèse distincte spécifiant pour cette présupposition propositionnelle particulière la teneur de la thèse $C$ :

THÈSE D : En accomplissant un acte de langage, un locuteur est engagé à avoir la croyance en l'existence de l'entité à laquelle il fait référence.

Un acte de langage et la dénégation de possession de l'état de croyance en l'existence de l'entité à laquelle il y est fait référence sont incompatibles. Leur accomplissement simultané serait paradoxal.

8. Les thèses $\mathrm{A}, \mathrm{B}, \mathrm{C}$ et $\mathrm{D}$ constituent la plate-forme théorique de l'élucidation de la problématique de l'engagement psychologique dans le langage suivant la perspective searlienne. Elles spécifient les états psychologiques qu'un locuteur est engagé à avoir (en première instance) du fait de son accomplissement d'un acte de langage.

Cette théorie de l'engagement psychologique est en mesure d'expliquer l'incompatibilité entre un acte de langage donné et la dénégation par le locuteur de sa possession de certains états mentaux. Elle a le pouvoir de rendre compte du caractère paradoxal de la combinaison d'une telle dénégation de possession d'un état psychologique et de l'acte de langage dont l'accomplissement engage le locuteur à avoir l'état en question.

Entre autres cas, notre théorie a le mérite d'expliquer la déficience formelle des situations d'illocution et de communication données en exemples en début d'article. En fait, la défectuosité de 
chacun des quatre doublets est élucidée par l'une des thèses qui viennent d'être formulées.

Le passant croisé dans la rue ne peut nous apprendre la nouvelle de la mort du Pape tout en nous indiquant ne pas croire au fait qu'il a existé parce que, ainsi que le spécifie la thèse $D$, il ne peut accomplir un acte de langage dans lequel il fait référence à une entité, ici le Pape, sans être engagé à avoir la croyance en l'existence de cette entité, croyance qu'il ne peut donc nier posséder.

De même, en vertu de la thèse $C$, le journaliste ne peut commenter le fait que Reagan a cessé de mentir en indiquant qu'il ne croit pas que le Président ait déjà menti. La proposition ayant pour teneur que Reagan a déjà menti est une présupposition propositionnelle de la proposition stipulant qu'il a cessé de mentir. L'acte de langage qui a cette dernière proposition comme contenu de représentation engage le locuteur à la possession de la croyance en cette présupposition propositionnelle. Le locuteur ne peut donc nier avoir la croyance que Reagan a déjà menti.

La franchise de l'homme politique qui, faisant une promesse, précise qu'il ne se croit pas capable de la remplir, sûrement fort louable moralement parlant, est toutefois catastrophique sur le plan logique. Suivant la thèse $B$, un locuteur est engagé à avoir la croyance en l'état de choses constituant la condition préparatoire de l'acte. Or, la capacité du locuteur d'effectuer l'action qu'il s'engage à accomplir est une présupposition illocutoire de son acte de promesse. Accomplissant cet acte de langage, notre politicien est engagé à avoir la croyance en sa capacité de la remplir et ne peut conséquemment pas dénier cette possession.

Finalement, c'est en vertu de la thèse $A$ que la vedette d'une réclame commerciale ne pourrait à la fois inciter à l'achat d'un produit et prétendre ne pas avoir le désir que le consommateur fasse cet achat. Le type d'incitation ici en cause est de nature directive. En ce sens, l'acte de langage accompli dans ou par un message publicitaire est assimilable à un ordre. Ce n'est évidemment pas un ordre mais à la façon d'un ordre il a pour objectif que l'allocutaire fasse l'action spécifiée. Comme un ordre, cet acte a pour condition de sincérité le désir du locuteur de voir l'allocutaire effectuer cette action. Un grand nombre de réclames publicitaires 
misent précisément sur ce désir, exprimé et donc supposé être effectivement possédé par une vedette ou une personnalité connue, de voir ceux à qui s'adresse le message se procurer l'objet « publicisé ». Selon la thèse $A$, un locuteur est engagé à la possession de l'état psychologique constitutif de la condition de sincérité de son acte de langage. La vedette de notre réclame ne peut donc nous inciter à acheter tel produit et dénier avoir le désir que nous le fassions puisque l'accomplissement de l'acte engage à la possession du désir.

9. Tous les cas d'illocution et de communication constitués, comme nos quatre exemples, d'un doublet dont le second terme est la dénégation de possession d'un état psychologique faisant l'objet d'un engagement dans l'accomplissement du premier acte de langage peuvent être adéquatement traités par notre théorie de l'engagement psychologique. C'est-à-dire qu'en mettant au jour ce à quoi les locuteurs sont engagés psychologiquement, cette théorie permet d'identifier les états mentaux qu'ils ne peuvent dénier posséder. Elle rend ainsi compte de l'incompatibilité entre les termes des doublets : de l'illogisme ou de la déficience formelle qui résulte de leur accomplissement simultané.

Trois remarques méritent d'être faites au sujet des thèses que nous proposons.

D'abord, telles qu'elles ont été ici introduites, les thèses A, B, $\mathrm{C}$ et $\mathrm{D}$ constituent les postulats de la théorie de l'engagement psychologique dans le langage. Il aurait été possible de donner à cette dernière un fondement axiomatique différent. En effet, nos thèses peuvent être élaborées suivant une autre stratégie théorétique adoptant comme point de départ la notion d'engagement illocutoire, c'est-à-dire entre actes de langage. Searle et Vanderveken caractérisent ainsi l'engagement illocutoire:

The idea behind the notion of illocutionary commitment is simply this : sometimes by performing one illocutionary act a speaker can be committed to another illocution. This can occur both in cases where the performance of one act by a speaker is eo ipso a performance of the other and in cases where the performance of the one is not a performance of the other and does not involve the speaker in a commitment to its explicit performance. ${ }^{13}$

13. Ibid., p. 23. 
Toutes les thèses de la théorie de l'engagement psychologique peuvent être fondées sur une relation d'engagement illocutoire à un acte de langage de la classe des expressifs, ces actes qui ont pour fonction l'expression d'états mentaux. Ainsi, l'engagement à avoir l'état psychologique constituant la condition de sincérité d'un acte - la thèse $\mathrm{A}$ - est " réductible » à l'engagement entre cet acte et l'acte expressif de l'état mental en question; de même, l'engagement à avoir la croyance en l'état de choses constituant la condition préparatoire d'un acte - notre thèse $B$ - est «réductible» ̀̀ l'engagement illocutoire de cet acte à l'acte expressif de cette croyance. Il en va de même pour les états psychologiques faisant l'objet des thèses $\mathrm{C}$ et $\mathrm{D}$. Tout engagement à la possession d'un état psychologique peut ainsi être ramené à un engagement illocutoire à l'acte expressif de l'état mental considéré. Par conséquent, l'identification d'une relation d'engagement entre une acte de langage $\mathrm{X}$ et un expressif permet la reconnaissance de l'engagement à la possession de l'état psychologique de l'expressif dans l'accomplissement de X. ${ }^{14}$

Second point qui n'est pas sans intérêt: les thèses de la théorie de l'engagement psychologique sont formalisables. Il est en effet tout à fait possible de leur donner une représentation symbolique apparentée à celle de la logique illocutoire de Searle et Vanderveken.

Troisième et plus importante remarque : il importe de préciser que les thèses $A, B, C$ et $D$ ne sont en fait que les constituants primitifs de la théorie de l'engagement psychologique et qu'elles n'éclairent que les cas les plus simples d'incompatibilité mettant en cause une dénégation de possession d'un état mental. À partir de ces thèses primitives, peuvent être construites de nouvelles thèses portant sur des cas plus complexes d'incompatibilité. Quelques-unes de ces thèses "dérivées " font ressortir de façon assez éclatante la pertinence philosophique de la problématique de l'engagement psychologique concomitant à l'accomplissement d'actes de langage. Aussi, en guise de conclusion, nous attarderons-nous

14. Par ailleurs, les états psychologiques qu'un locuteur est engagé à avoir peuvent également faire l'objet d'un acte de type déclaratif, catégorie dont le but illocutoire est de rendre existant, par le fait même de l'énonciation, l'état de choses correspondant au contenu propositionnel. Un locuteur peur se déclarer engagé à la possession d'un état mental. 
quelque peu sur trois d'entre elles, simplement en les présentant sans les fonder dans une relation d'engagement illocutoire.

La première thèse complexe à considérer est la suivante:

THĖSE E : En accomplissant un acte de langage qui l'engage à avoir un premier état psychologique, un locuteur est par le fait même engagé à avoir la croyance qu'il possède ce premier état mental.

Engagé à avoir un état psychologique en vertu de son accomplissement d'un acte de langage, un locuteur est du même coup également engagé à avoir la croyance qu'il possède le premier état mental. Il ne lui est ainsi pas possible de nier avoir cette croyance. Par exemple, engagé à avoir l'intention d'effectuer la chose promise, le locuteur qui promet est aussi engagé à croire qu'il a cette intention et il ne peut dénier avoir également la croyance qu'il a cette intention. Il serait paradoxal qu'un locuteur affirme «Je ne crois pas que j'ai l'intention de venir» simultanément à son accomplissement de l'acte « Je promets de venir ». Un engagement à la possession de la croyance qu'on le possède est donc concomitant à l'engagement à avoir un état psychologique. Engageant à la possession d'un état mental, l'accomplissement d'un acte de langage engage par le fait même le locuteur à avoir la croyance qu'il le possède.

Cette thèse vaut pour tous les états psychologiques faisant l'objet d'un premier engagement. Elle peut donc recevoir une formulation spécifique relativement à chacun des états mentaux spécifiés dans les thèses $A, B, C$ et $D$. On dira donc globalement qu'en accomplissant un acte de langage, un locuteur est engagé à avoir les croyances qu'il possède:

a) l'état psychologique constitutif de la condition de sincérité de l'acte;

b) la croyance en l'état de choses constituant la condition préparatoire de l'acte;

c) la croyance en l'état de choses correspondant à la proposition qui est une présupposition propositionnelle de l'acte ;

d) et la croyance en l'existence de l'entité à laquelle il est fait référence. 
Ainsi, (a) l'accomplissement d'une assertion engage le locuteur à avoir la croyance en sa possession de la croyance en la teneur de l'assertion, celui d'une promesse l'engage à avoir la croyance en sa possession de l'intention de faire la chose promise et celui d'un ordre à avoir la croyance en sa possession du désir que l'allocutaire exécute l'action ordonnée. De même, (b) en accomplissant un ordre, un locuteur est engagé à avoir la croyance qu'il possède la croyance que l'allocutaire est capable d'effectuer cet ordre. Pareillement, (c) l'accomplissement de l'assertion affirmant le retour d'Europe de Pierre engage le locuteur à avoir la croyance qu'il possède la croyance que Pierre était en Europe et (d) l'accomplissement de l'assertion "Jean fume beaucoup " l'engage à avoir la croyance qu'il a la croyance en l'existence de Jean.

Suivant donc la thèse $\mathrm{E}$, l'engagement à la possession d'un premier état psychologique contracté à l'occasion de l'accomplissement d'un acte de langage implique l'engagement à avoir le second état mental qu'est la croyance en la possession du premier. Un locuteur qui est engagé à avoir un état psychologique est ipso facto engagé à avoir la croyance qu'il le possède.

Or, cet autre état de croyance tombe lui aussi sous le coup de la thèse $\mathrm{E}$ : engagé à avoir la croyance de deuxième ordre qu'il possède un état psychologique qu'il est initialement engagé à avoir, le locuteur est par le fait même engagé à a voir la croyance de troisième ordre qu'il possède la croyance de deuxième ordre. À la suite, la croyance de troisième ordre implique l'engagement à avoir une croyance de quatrième ordre et ainsi de suite indéfiniment.

La thèse $\mathrm{E}$ donne donc lieu à une régression à l'infini de croyances relatives à la possession d'un état psychologique qu'un locuteur est engagé à avoir en première instance du fait de son accomplissement d'un acte de langage. Ce trait est suffisamment remarquable pour faire l'objet d'une thèse particulière:

THÈSE F : En accomplissant un acte de langage qui l'engage à avoir un premier état psychologique, un locuteur est par le fait même engagé à avoir une infinité de croyances relatives à sa possession de ce premier état mental.

Comme la thèse $\mathrm{E}$, la thèse $\mathrm{F}$ s'applique eu égard aux différents états psychologiques identifiés par les thèses primitives 
A, B, C et D. Elle peut donc être particularisée pour chacun de ces états. Ces différentes spécifications conduisent à la considération générale suivante : en accomplissant un acte de langage, un locuteur est engagé à avoir :

- une infinité de croyances relatives à sa possession de l'état psychologique constitutif de la condition de sincérité de l'acte;

- une infinité de croyances relatives à sa possession de la croyance en l'état de choses constitutif de la condition préparatoire de l'acte ;

- une infinité de croyances relatives à sa possession de la croyance en l'état de choses correspondant à la proposition qui est une présupposition propositionnelle de l'acte;

- et une infinité de croyances relatives à sa possession de la croyance en l'existence de l'entité à laquelle il est fait référence.

Un locuteur serait de la sorte engagé à la possession de «quatre infinités » distinctes (au moins) d'états psychologiques.

L'idée de l'engagement à la possession d'une infinité d'états mentaux n'est pas triviale: on ne s'attendrait pas intuitivement à ce qu'un locuteur soit engagé du fait de son accomplissement d'un acte de langage à avoir une série infinie d'états psychologiques.

Par ailleurs, d'aucuns voudraient peut-être contester la validité de cette thèse en invoquant l'argument de la limitation du cerveau des sujets parlants, limitation qui bien sûr les empêcherait de posséder une infinité d'états psychologiques. Il faut à ce propos préciser que la thèse $F$ ne stipule absolument pas qu'un locuteur possède une infinité d'états mentaux mais plutôt qu'il est engagé à cette possession. Or, ainsi que nous avons déjà eu l'occasion de le faire remarquer, l'engagement à avoir un état psychologique n'en implique pas la possession effective. Il est tout à fait possible à un locuteur d'être engagé à avoir un état mental sans du tout le posséder. Et donc, un locuteur peut se trouver engagé à la possession d'une infinité de croyances relatives à sa possession d'un état psychologique qu'il possède ou non cet état et cette infinité de croyances. Même en ne les possédant pas, le locuteur serait malgré tout engagé à avoir l'état mental et l'infinité de croyances relatives à cette possession. 
La troisième thèse complexe de nature assez exceptionnelle est celle-ci :

THÈSE G : En accomplissant un acte de langage qui l'engage à avoir un premier état psychologique, un locuteur est par le fait même engagé à avoir la croyance en sa propre existence.

Un locuteur est engagé à la possession de la croyance qu'il existe dès lors qu'il accomplit un acte de langage où il réfère à luimême. Cette autoréférence est effectuée dans un grand nombre d'actes de langage de différentes sortes. Par exemple, je réfère à ma propre personne dans l'assertion «Je termine bientôt cet article !». Le locuteur réfère également à lui-même dans tous les actes de la classe des commissifs, comme la promesse, dans lesquels il s'engage à effectuer une action donnée. Suivant notre thèse $\mathrm{D}$, en accomplissant de tels actes de langage, le locuteur est engagé à a voir la croyance en son existence puisqu'il est lui-même l'entité à laquelle il fait référence.

La thèse $\mathrm{G}$ a une portée beaucoup plus générale. Elle stipule que l'accomplissement de tout acte de langage engage le locuteur à la possession de la croyance qu'il existe du fait qu'il l'engage d'abord à avoir un premier état psychologique.

Car de même que cet engagement à la possession d'un premier état psychologique corrélatif à l'accomplissement d'un acte de langage va de pair avec l'engagement à avoir la croyance en la possession de cet état mental, il est également concomitant à l'engagement, par le locuteur, à la possession de la croyance en sa propre existence. Tout locuteur qui accomplit un acte de langage (standard) est ainsi automatiquement engagé à avoir la croyance qu'il existe. Et cela précisément parce qu'il est engagé à avoir un premier état psychologique en accomplissant son acte de langage. Par exemple, un locuteur qui accomplit un acte de promesse est initialement engagé à avoir l'intention d'effectuer la chose promise et de ce fait engagé à avoir la croyance en sa propre existence. Son engagement à la possession de l'intention l'engage à croire qu'il existe; car à défaut d'exister le locuteur ne pourrait avoir cette intention. Il n'est donc pas possible au locuteur de promettre et de dénier avoir la croyance en son existence pas plus qu'il ne lui est possible de dénier avoir l'intention de remplir sa promesse. Cette promesse est incompatible avec l'acte de dénégation de la possession 
de la croyance du locuteur en son existence. Il ne peut, dans le même contexte d'énonciation, accomplir l'acte «Je promets de venir » et affirmer "Je ne crois pas que j'existe».

L'accomplissement d'un acte de langage, parce qu'il engage le locuteur à la possession d'un premier état mental, l'engage aussi à avoir la croyance qu'il existe. L'existence est en quelque sorte prérequise à la possession d'états psychologiques et à l'engagement à avoir ces états mentaux. Celui qui est engagé à la possession d'un état psychologique du fait de sa performance langagière est ainsi par le fait même engagé à croire en sa propre existence. L'engagement psychologique dans le langage implique donc un cartésianisme linguistique: "Je parle, donc je suis ». Cette nouvelle version du cogito, suivant en cela une tendance contemporaine (bonne ou mauvaise?) de la philosophie, le ferait passer de l'ordre de la pensée à celui du dire.

Département d'information et de communication

Université Laval 